\title{
Thermodynamics of Geysers: Application to Titan
}

\author{
Ralph D. Lorenz \\ Lunar and Planetary Laboratory, University of Arizona, Tucson, Arizona 85721 \\ E-mail: rlorenz@1pl.arizona.edu
}

Received May 11, 2001; revised October 5, 2001

\begin{abstract}
General constraints on geyser phenomena are developed and applied to speculative methane geysers on Titan. Variation of boiling point with depth of ethane-methane-nitrogen fluids is found to be of order $0.3 \mathrm{~K} \mathrm{~m}^{-1}$, in contrast with around $2 \mathrm{~K} \mathrm{~m}^{-1}$ for water on Earth. It is found that geysers are possible on Titan but require enhancements of $\sim \mathbf{1 0 0}$ above global-average geothermal heat flux, a factor similar to that required on Earth. Eruption velocities of order $25 \mathrm{~m} \mathrm{~s}^{-1}$ appear to be typical for 10-m-deep geyser vents on both Earth and Titan. While eruption velocities on Earth are usually limited by the low sound speed in water-steam mixtures, sound speeds in Titan fluids are higher and the Titan limit is imposed by the available energy in the boiling fluid. Eruption intervals should be nearly the same for geysers with equivalent plumbing. There is an interesting symmetry between geysers on Titan and those on Earth: The volatility of the relevant fluid scales with the available heat flow. (c) 2002 Elsevier Science (USA)

Key Words: Titan; geological processes; volcanism.
\end{abstract}

\section{INTRODUCTION}

The motion of liquid water on the Earth's surface presents some of the planet's most interesting phenomena. Among the most spectacular of these are geysers, sporadic or periodic geothermally driven eruptions of water jets reaching tens of meters into the sky. Saturn's giant moon Titan has a thermodynamic surface environment that allows liquid hydrocarbons, and it may be that geysers are among the phenomena that these liquids exhibit.

In this paper simple constraints on the circumstances that permit and control geyser eruption are elaborated. These are then applied to hydrocarbons on Titan, and they show that classic geyser eruption is certainly plausible on Titan. Some additional, more exotic, geyser mechanisms are considered, and finally prospects for observing such phenomena with Cassini are considered.

In addition to the prediction of the characteristics of geysers on Titan per se, this exercise illustrates the key aspects of geysers, in perhaps a simpler way than has been heretofore presented.

\section{GEYSER PHENOMENA}

As background to speculation about Titan, I briefly summarize here the five known geyser-like phenomena observed in the Solar System. Only the two terrestrial phenomena are really geyser- like; the others are arguably more representative of explosive maar-style volcanism than geysers per se but are included to underscore the wide range of dynamic behavior of volatiles in the Solar System.

The first type is the canonical geyser, named after the archetype "Geysir" in Iceland. Rinehart (1980) presents a useful overview of geysers, describing some model investigations. A paper with illuminating detail, but perhaps too much for the present application, is that by Dowden et al. (1991). The paper by Kieffer (1982) is also particularly recommended for hydrodynamic and thermodynamic detail. Understanding of geysers can be aided by laboratory-scale physical models (e.g., Anderson 1978).

In a classical geyser, a column of water in a plumbing system with some vertical extent (see Fig. 1) is heated geothermally. Since at a given depth in the column, the local pressure is higher than atmospheric because of the hydrostatic head of the water above, the local boiling point is higher than that at the surface. If, as the column heats up, some point at depth reaches the local boiling point, bubbles of vapor begin to form. These may intially encourage local convection, transporting heat to cooler parts of the column before the vapor collapses back to liquid. More and more of the column therefore gets closer and closer to the boiling point. Eventually the whole column boils instantaneously, usually because the growing bubble volume pushes some water out of the top of the column and thereby unloads it. The loss of hydrostatic head drops the pressure in the column, so that the pressure becomes less than the local saturation vapor pressure over a large fraction of the column.

In well-behaved geysers, where the subsurface plumbing is recharged at a constant rate and is sufficiently insulated from surface conditions so that local meteorology does not affect the loss of heat from the water column, geysers have a fairly constant interval between eruptions. Old Faithful in Yellowstone National Park, USA, is the usual example. Wide-throated geysers with surface pools, such as Strokkur in Iceland, are more susceptible to nondeterministic variations in surface heat loss and so are more pseudoperiodic. Where geysers are wide-throated compared with the volume of the plumbing system, the eruption will be short and violent. Other geysers, such as Old Faithful, with a constricted exit, will have a longer lasting "firehose" style of eruption. 


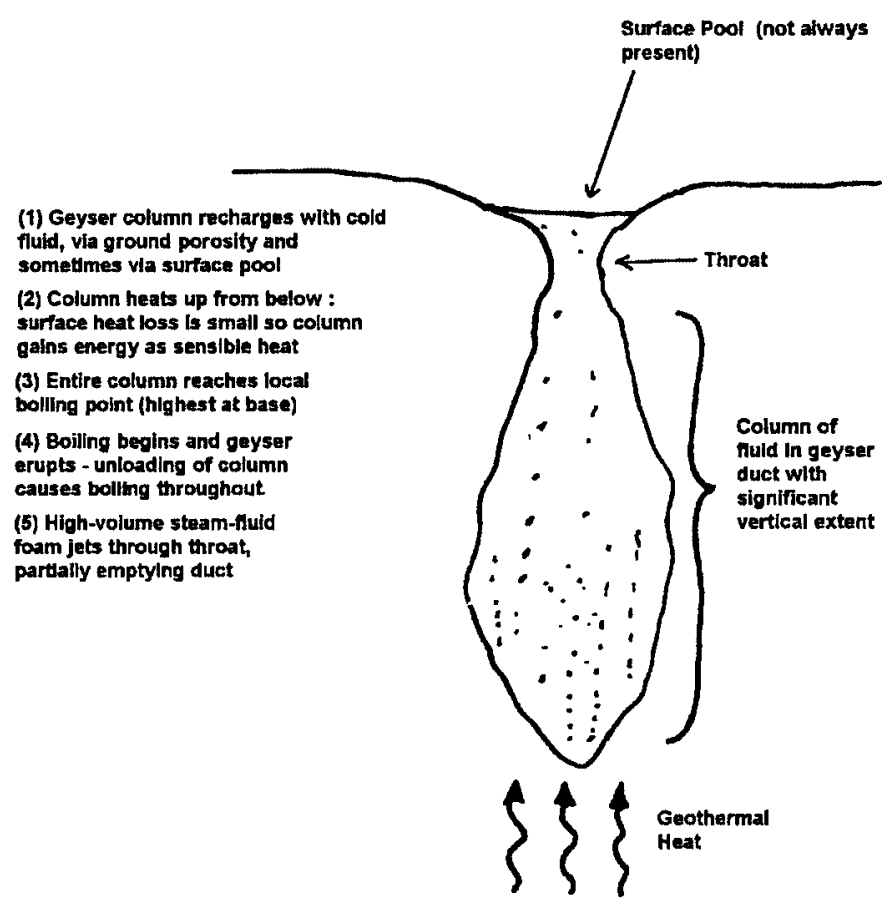

FIG. 1. Schematic of a geyser and the steps in geyser eruption.

Addition of soap is a well-known method of stimulating the eruption of an otherwise marginal geyser. Several mechanisms may be at work. First, addition of soap reduces surface tension and may therefore stimulate bubbles to form. Second, soap flakes may act as nuclei for bubbles. Finally, formation of a surface foam may insulate the water column and thereby allow it to warm to the boiling point (if there is significant heat loss at the surface which would otherwise prevent it from doing so) and this frothing at the mouth may unload the column and hence stimulate boiling.

The classic boiling water geyser described above relies on the volume change from liquid to vapor. An alternate mechanism arises from volume change due to exsolution of dissolved gasses-a phenomenon well known to anyone who has opened a bottle of beer. The most (in)famous example is the catastrophic eruption of Lake Nyos in Cameroon (e.g., Clarke 2001, Zhang 1996).

Lake Nyos receives a continuous addition of volcanic gasesprincipally $\mathrm{CO}_{2}$ - at its base, without large addition of volcanic heat. The $\mathrm{CO}_{2}$ dissolves in the cold bottom waters and remains at depth, with only modest mixing up to the surface. Eventually, a significant fraction of the water becomes saturated with $\mathrm{CO}_{2}$, creating a metastable situation analogous to a thermal geyser being at the threshold of boiling. Addition of more $\mathrm{CO}_{2}$, or displacement of saturated liquid to shallower depths, by winddriven disturbance, for example, causes bubbles to form. These bubbles ascend, and in so doing drag the surrounding liquid to shallower depths. Here the lower hydrostatic pressure allows more bubbles to form and the process accelerates. The ascending and expanding foam column breaks the surface and fountains out.

During the eruption of Nyos in 1986, the large volume of $\mathrm{CO}_{2}$ released flowed as a contiguous cloud and suffocated the population of a village a few kilometers down valley from the lake, killing some 1700 people. The eruption occurred at night and was not witnessed, but the distribution of dead animals thrown from the eruption placed a lower limit of $120 \mathrm{~m}$ on the eruption height. Simulations (Zhang 1996) indicate that from an eruption depth of $150 \mathrm{~m}$, the eruption velocity would be about $70 \mathrm{~m} / \mathrm{s}$, with $\mathrm{CO}_{2}$ reaching a maximum altitude of some $250 \mathrm{~m}$.

The geysers observed on Neptune's moon Triton (Soderblom et al. 1990, Kirk et al. 1995) are mentioned here more for completeness than phenomenological equivalence and in particular as a caution that one must not equate a cold, low-energy environment with an absence of energetic phenomena. Briefly, Voyager 2 cameras observed dark vertical plumes ascending some $10 \mathrm{~km}$ into the thin $(17-\mu \mathrm{bar})$ nitrogen atmosphere. Since the surface temperature of Triton $(40 \mathrm{~K})$ is below the freezing point of nitrogen, it is believed that these plumes result from the pressurization of subsurface reservoirs of nitrogen by local heating and the subsequent venting of these reservoirs through fractures in the nitrogen ice surface. The steep saturation vapor pressure curve of nitrogen at these temperatures means that only a small elevation in temperature is required to obtain overpressure sufficient to achieve plume exit velocities adequate to reach $8-\mathrm{km}$ altitude and to raise a $\sim 4-\mathrm{m}$-thick layer of ice. Although internal heating is quite possibly a factor (e.g., Duxbury and Brown 1997), the association of the observed plumes with the subsolar point suggests that heating by a solid-state greenhouse on Triton is a principal factor.

The fourth phenomenon, observed on the jovian moon Io, is the plumes of $\mathrm{SO}_{2}$, most spectacularly that associated with the volcanic structure Prometheus. These plumes have a beautiful geometric shape some $300 \mathrm{~km}$ in diameter owing to the expansion into vacuum. Kieffer (1982) provides a detailed discussion. In addition to these visible plumes, there are probably many "stealth" plumes, where the pressure-temperature evolution of the expanding plume does not fall below the condensation point and thus no snow particles form to make the plume optically detectable.

A fifth phenomenon, which is perhaps not yet mature enough in its study to be considered a robust example, is that of the curious features observed on the martian polar cap, the so-called dark spiders. These are believed to be deposits of dark dust ejected from holes in the slab of $\mathrm{CO}_{2}$ frost.

The latter three examples probably have more in common with maar eruptions on Earth, in that the volatiles are confined by the strength of the surface rock, rather than the hydrostatic head of liquid above the boiling region. However, they illustrate the ubiquity of dynamic phenomena in the Solar System: The relevant working fluids show a consistent trend of volatility with distance from the Sun, namely $\mathrm{H}_{2} \mathrm{O}, \mathrm{CO}_{2}, \mathrm{SO}_{2}$, and $\mathrm{N}_{2}$. The factors and processes that select the materials for the 
outer envelopes of planets ensure a certain level of volatility, perhaps providing the most interesting Solar System that one can construct. As an aside, this trend is interesting in the sense that investigations of zonal climate (Lorenz et al. 2001) suggest that many planetary atmospheres may seek states of maximum entropy production: Zonal advective heat transport appears to scale not with pressure, as is often assumed, but with the radiative setting, determined ultimately by distance from the Sun.

\section{A SIMPLE CONDITION FOR GEYSER ERUPTION}

It is clear that the driving force for classic geysers is the boiling of water. It follows, therefore, that somehow the liquid must be brought to the boiling point. This is why geysers are associated with regions of exceptional geothermal heat flow.

A homogenous layer of ice or rock will have a temperature profile $T(z)$ that is essentially linear, with a slope $d T / d z$ equal to the geothermal heat flux divided by the thermal conductivity of the rock. If a static column of material is inserted into the rock, and that material has a different effective thermal conductivity, then the local temperature profile will be perturbed; a metal rod, for example, or a convecting column of liquid, may act as a thermal short circuit and reduce the local $d T / d z$. However, if the column is sufficiently long and narrow, the perturbation will be small and the column will assume the $d T / d z$ of the surrounding rock. On both Earth and Titan, the thermal conductivity of the geyser fluid is several times lower than that of the surrounding rock. A narrow water column will therefore asymptotically warm to this ambient temperature profile.

More generally, if the column is not so narrow as to completely suppress convection, then the column will deviate from the bedrock's natural temperature profile. The surface temperature of the column (the "pool temperature") will depend both on the strength of convection, i.e., the effective thermal conductivity of the column, and on the effectiveness of surface heat transfer between the top of the column and the atmosphere. The low-conductivity, high-surface-transfer limit would have a pool temperature $T_{\mathrm{o}}$ close to surface ambient $T_{\mathrm{S}}$ and a depth profile $d T / d z$ equal to that of the surrounding rock, as discussed above. Another limit is a "high-conductivity" (vigorously convecting) column with a high surface heat transfer. Such a column will be nearly isothermal at the surface temperature. This situation, resembling that of most terrestrial lakes, is not of particular interest and pertains to hot pools, mudpots, etc. A highconductivity, low-surface-heat-transfer situation, however, will be nearly isothermal, but at a temperature close to that at the bottom of the column. This situation, with a column that is sufficiently deep and/or hot, presents the most favorable circumstances for geyser eruption. A low-conductivity, low-surfaceheat-transfer situation will initially begin at the ambient profile, but as the root begins to boil, convection will be enhanced and the system will evolve into a high-conductivity one until the whole column lies on the boiling line and the geyser erupts.

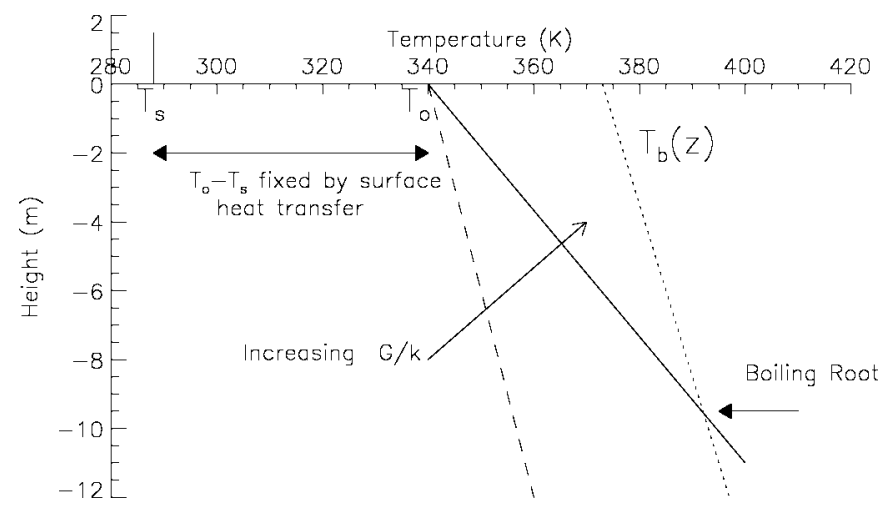

FIG. 2. Schematic of subsurface temperature gradients. If the ambient geotherm (e.g., dashed line) is steeper, i.e., less increase of temperature with depth, than the boiling point curve (dotted line) then geysering will never occur. If, however, high heat flow causes it to be steep, it will intersect the boiling point curve and boiling will begin at the root. This enhances heat transport in the geyser, bringing the surface geyser temperature $T_{\mathrm{o}}$ from the initial ambient $T_{\mathrm{s}}$ to the local boiling. Once a large part of the column is at the boiling point, the geyser can erupt.

If we consider the spectrum of possibilities above in a more quantitative manner, sooner or later the liquid column of depth $h$ will acquire a temperature profile $T(z)=T_{\mathrm{o}}+G z / k$, where $G$ is the geothermal heat flux and $k$ is the effective conductivity (including convective transfer) of the geyser column. The narrow plumbing case (low conductivity, high surface transfer) has $T_{\mathrm{o}}=$ $T_{\mathrm{s}}$ and $k$ is the thermal conductivity of the surrounding bedrock. The hot-column (high-conductivity, low-surface-transfer) limit has $T_{\mathrm{o}} \gg T_{\mathrm{s}}$ but a high effective $k$.

These temperature profiles can then be compared with the boiling point as a function of depth $T_{\mathrm{b}}(z)$; a crucial element of the geyser instability is the increase in boiling point with depth. Although this function is in general nonlinear, for small distances we can consider a linear boiling point slope, which is about $2 \mathrm{~K} \mathrm{~m}^{-1}$ for water on Earth.

Clearly, whatever the surface temperature offset from the surface boiling point, if $d T_{\mathrm{b}} / d z>G / k$, the column will never reach boiling anywhere (see Fig. 2) and geysers will not occur. For $d T_{\mathrm{b}} / d z=2 \mathrm{~K} \mathrm{~m}^{-1}$, it follows that $G / k$ must exceed this value where geysers occur. Taking terrestrial average values, a conductivity of rock of $2 \mathrm{~W} \mathrm{~m}^{-1} \mathrm{~K}^{-1}$ and $\mathrm{G}=60 \mathrm{~mW} \mathrm{~m}^{-2}$, we have $G / k=0.03 \mathrm{~K} \mathrm{~m}^{-1}$ and obtain the reassuring if unexciting result that in general geysers cannot occur on Earth (as, indeed, in general they do not). However, wherever the geothermal heat flow is enhanced by a factor of 50-100, geysers can occur.

\section{THERMODYNAMIC SETTING ON TITAN}

Titan represents a unique environment. While in some respects the Io and Triton cases may be considered near-equivalent in that they both have very thin atmospheres and low gravities, Titan is distinct and more Earthlike in that the surface atmospheric pressure is significant. 
The low gravity has two effects. One is that a plume with a given exit velocity will reach a higher altitude. The other is that the variation of pressure with depth in the geyser column, and thus the increase of boiling point with depth, is low.

It may be assumed that the saturation vapor pressure of surface liquids on Titan is at, or is slightly higher than, the local partial pressures of those gases, namely nitrogen and methane. Climate models and interpretations of Voyager data usually rely on the assumption of thermodynamic equilibrium, i.e., that the atmospheric partial pressures are equal to the saturation values. On the Earth, this assumption is not quite true for water vapor, which is often advected away from the thin boundary layer over wet surfaces.

The dependence of saturation vapor pressure on temperature can be crudely parameterized by fitting the results of the multicomponent nonideal solubility model used in Lorenz et al. (1999) and McKay et al. (1993) based on material in Kouvaris and Flasar (1991), Thompson et al. (1992), and Thompson (1985).

Specifically, if a volatile inventory comprising 7 bar of ethane (a reasonable estimate of the amount produced by photolysis over the age of the Solar System; see, e.g., Yung et al. 1984), 1.8 bar of nitrogen, and 1 bar of methane is deposited on Titan, it would yield an atmosphere at $94.3 \mathrm{~K}$ of 1.438 bar $\mathrm{N}_{2}$ and 0.05 bar $\mathrm{CH}_{4}$, or a pressure of 1.49 bar and a methane humidity of about $60 \%$. Such an atmosphere is compatible with Voyager observations (see, e.g., Lorenz et al. 1999).

The model, and indeed the data used to fit it, is not accurate to the number of significant digits shown in absolute terms, but the important point here is the dependence of pressure with temperature, where the model is quite adequate. For a temperature of $95.3 \mathrm{~K}$, the corresponding pressure is $1.515 \mathrm{bar}$, and for a temperature of $96.3 \mathrm{~K}$, it is $1.541 \mathrm{bar}$. Thus, the saturation pressure increases by about $0.03 \mathrm{bar} / \mathrm{K}$. This latter slope is more or less the same as for pure methane.

Titan surface liquids probably have a density of around $600 \mathrm{~kg} \mathrm{~m}^{-3}$ (since the densities of liquid methane, ethane, and nitrogen are 450,650 , and $800 \mathrm{~kg} \mathrm{~m}^{-3}$ respectively) and the ambient gravity is $1.3 \mathrm{~m} / \mathrm{s}$, so the hydrostatic pressure of a liquid column is a factor of 10 lower on Titan than on Earth, specifically $9 \mathrm{mbar} \mathrm{m}^{-1}$, compared with $100 \mathrm{mbar} \mathrm{m}^{-1}$ for water on Earth.

A column of liquid on the threshold of boiling will have a temperature variation with depth that is found by the boiling temperature of the local (atmospheric + hydrostatic) pressure. The result is about $0.3 \mathrm{~K} / \mathrm{m}$. This may be compared with the corresponding result for water on Earth of about $2 \mathrm{~K} / \mathrm{m}$.

On Titan, the heat flux is $5 \mathrm{~mW} \mathrm{~m}^{-2}$, assuming all the geothermal heat flow is due to a chondritic core (tidal dissipation is likely to be an order of magnitude smaller). The thermal conductivity of the surface at $94 \mathrm{~K}$ is around $5 \mathrm{~W} \mathrm{~m}^{-1} \mathrm{~K}^{-1}$ if the crust is water ice; other ices are of lower thermal conductivity (ammonia-rich water ice has a thermal conductivity of about $1 \mathrm{~W} \mathrm{~m}^{-1} \mathrm{~K}^{-1}$ at this temperature; Lorenz and Shandera 2001) and thus the average geothermal gradient is likely to be $0.001-$ $0.005 \mathrm{~K} \mathrm{~m}^{-1}$, or two orders of magnitude too low to exceed the boiling point.

Hence, for geysers to occur on Titan, an enhancement of the geothermal heat flow of about a factor of 100 is required, the same factor as required on Earth. Clearly, Titan's interior structure is not well known, and thus the heterogeneity of its internal heat flow and the likelihood of such enhancements cannot be assessed. Crustal thinning over a mantle plume, the presence of intruded magma in the crust, and perhaps buried heat in an impact melt pool are all possible mechanisms for enhancing local heat flow.

\section{LIMITS ON GEYSER ERUPTION VELOCITY}

Eruption velocity depends on many factors, most especially on the depth of the plumbing and the associated water superheat. Although there are some exceptional examples such as Waimangu, which reached $450 \mathrm{~m}$ in 1904 (Rinehart 1980), and Excelsior in Yellowstone, which exceeded $100 \mathrm{~m}$ in the $1880 \mathrm{~s}$, in general geysers reach a few tens of meters. Let us therefore take $30 \mathrm{~m}$ as a typical number. The required eruption velocity is therefore of order $(2 \times 30 \times 9.81)^{0.5}$, or $25 \mathrm{~m} \mathrm{~s}^{-1}$. We take, without further justification, a depth scale for the geyser root of $10 \mathrm{~m}$.

\subsection{Energy Limit}

The driving force is the sensible heat in the water column associated with the temperature excess above the surface boiling point. For water, the excess is $c_{p} \Delta T$, or $4200 \Delta T \mathrm{~J} \mathrm{~kg}^{-1}$, or for $\Delta T=20 \mathrm{~K}$ it is equivalent to the kinetic energy of water at some $400 \mathrm{~m} \mathrm{~s}^{-1}$. (Equivalently, a water jet decelerated from $400 \mathrm{~m} \mathrm{~s}^{-1}$ by friction would increase in temperature by only $20 \mathrm{~K}$.)

Many works on geysering associate the kinetic energy of the erupting plume with this energy excess (e.g., Rinehart 1980). While this is indeed easy to understand, it is not a powerful limit and substantially overestimates real geyser performance, where heights rarely exceed a few tens of meters. As we shall see, the efficiency with which the geyser machine can convert heat into work is rather modest.

For convenience, we consider the most energetic part of the geyser eruption, that corresponding to the root of the eruption, where the local temperature $T_{\mathrm{R}}$ first exceeds the local boiling point. The column will on average be at a temperature intermediate between that and the surface boiling point $T_{\mathrm{B}}$.

\subsection{Work Limit}

Only by expanding into steam can the fluid in the geyser perform mechanical work. If the geyser were an ideal thermodynamic engine, it would have a limiting (Carnot) efficiency $\eta$ of $\left(1-T_{\mathrm{B}} / T_{\mathrm{R}}\right)$ or $\Delta T / T_{\mathrm{R}}$. The mechanical energy available per kilogram of fluid would be $c_{p} \Delta T\left(1-T_{\mathrm{B}} / T_{\mathrm{R}}\right)$. For a geyser with a root at a depth of $10 \mathrm{~m}$ on Earth, we have $\Delta T \sim 20 \mathrm{~K}$, $T_{\mathrm{R}} \sim 393 \mathrm{~K}$, and thus $\eta \sim 5 \%$. For the case above, the maximum 
launch velocity is thus a more reasonable $90 \mathrm{~m} \mathrm{~s}^{-1}$, allowing the plume to reach a height of $400 \mathrm{~m}$.

Imagine a $10 \mathrm{~m}$-deep vent on Titan: The boiling temperature at its base would be $3 \mathrm{~K}$ higher than the surface temperature, and assuming a $c_{p}$ of $3000 \mathrm{~J} \mathrm{~kg}^{-1} \mathrm{~K}^{-1}$, there is about $300 \mathrm{~J} \mathrm{~kg}^{-1}$ of work available for this deepest parcel of fluid-enough to hoist the liquid some $230-\mathrm{m}$ high, or launch it at a velocity of $25 \mathrm{~m} \mathrm{~s}^{-1}$. We may note that the conversion efficiency on Titan is about half that on Earth since although the temperature is a factor of 3 lower, the temperature differences driving activity are about seven times smaller than on Earth.

It is seen that the specific available energy of the eruption depends quadratically on the degree of superheat $\Delta T$. In the formalism above, it appears once in the sensible heat and once in the conversion efficiency.

In passing, it is worth noting that Kirk et al. (1995) present an approximate expression for the eruption velocity $u$ of so-called high-entropy or condensing geysers, where $u \sim\left(2 L \Delta T / T_{\mathrm{B}}\right)^{0.5}$. Although more elaborately derived, this expression states in essence that all of the plume begins as vapor and gives up all of its latent heat, which is then converted into kinetic energy with the maximum possible (Carnot) efficiency.

\subsection{Sonic Limit}

As noted by Kieffer (1977), the flow in energetic geysers is often hydrodynamically choked; that is, flow is constrained to the local sound speed at the narrowest part of the geyser duct, the "throat". For pure liquids or gases this is rarely a limitation in nature (although for the latter, it is fundamental in the design of jet and rocket engines.) However, mixtures of liquids and gases tend to have low sound speeds, since the sound speed is essentially the square root of the ratio of bulk modulus to density. To a first order the density is closer to that of the liquid than to that of the gas. However, the compressibility of the foam is essentially that of the gas. Thus the sound speed of the mixture is quite low.

An elaborate formalism for computing sound speed $c$ is given in Kieffer (1977), but a more convenient and sufficiently accurate equation, as given by Wood (1941), is $c=(A B)^{-1}$, where

$$
A=\left[\alpha \rho_{\mathrm{g}}+(1-\alpha) \rho_{\mathrm{l}}\right]^{0.5}
$$

and

$$
B=\left[\left(\frac{\alpha}{\rho_{\mathrm{g}} c_{\mathrm{g}}^{2}}\right)+\left(\frac{1-\alpha}{\rho_{1} c_{1}^{2}}\right)\right]^{0.5},
$$

in which $\alpha$ is the void (or gas volume) fraction, $\rho$ is the density of each phase, and subscripts $g$ and 1 denote the gas and liquid, respectively.

The speeds of sound in water and steam at the boiling point at 1 bar are 446 and $1500 \mathrm{~m} \mathrm{~s}^{-1}$, respectively. For Titan, the speed of sound in gaseous nitrogen at $94 \mathrm{~K}$ is about $200 \mathrm{~m} \mathrm{~s}^{-1}$; in gaseous methane ("steam" on Titan) it would be around $250 \mathrm{~m} \mathrm{~s}^{-1}$

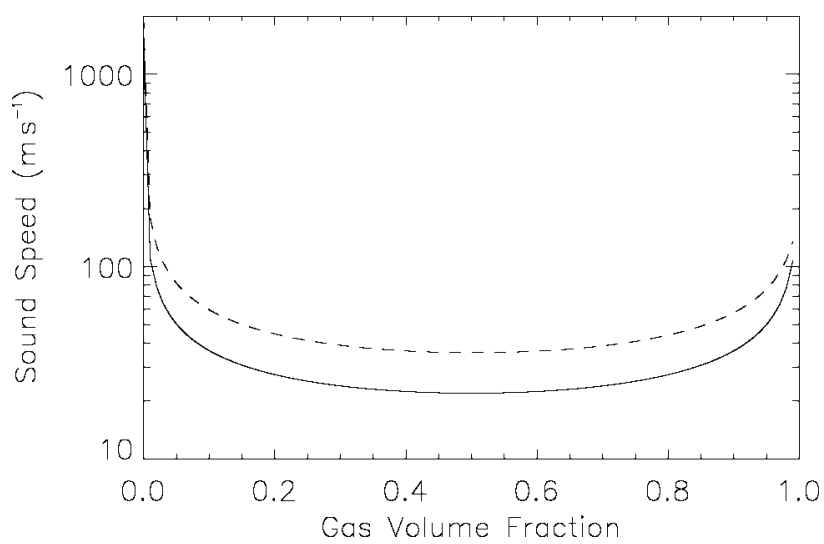

FIG. 3. Dependence of sound speed of a two-phase mixture on void fraction. Water steam is shown as a solid line; methane-rich Titan fluids are shown by a dashed line. Titan speeds are in general higher, except when the mixture is almost all gas.

(since methane condenses at 1 bar above this temperature, the sound speed is rarely measured directly but is extrapolated from higher temperatures). Liquid methane and ethane at $95 \mathrm{~K}$ have sound speeds of 1500 and $2000 \mathrm{~m} \mathrm{~s}^{-1}$, respectively (e.g., Garry 1986.)

The speeds of sound for liquid-steam mixtures for Earth and Titan are plotted in Figs. 3 and 4. Sound speeds are generally (except for very high gas fractions) somewhat higher for Titan than for Earth.

The maximum steam mass fraction $f$ is given simply by $c_{p} \Delta T / L$, or $0.0018 \Delta T$, due to water's very high latent heat of vaporization. The same calculation for Titan yields a rather higher amount, $0.006 \Delta T$. For a given $\Delta T$, Titan has a steam fraction three times higher, but for a given depth (with a six times smaller $\Delta T$ ), Titan's steam fraction would be half as much. In each case, $f \ll 1$.

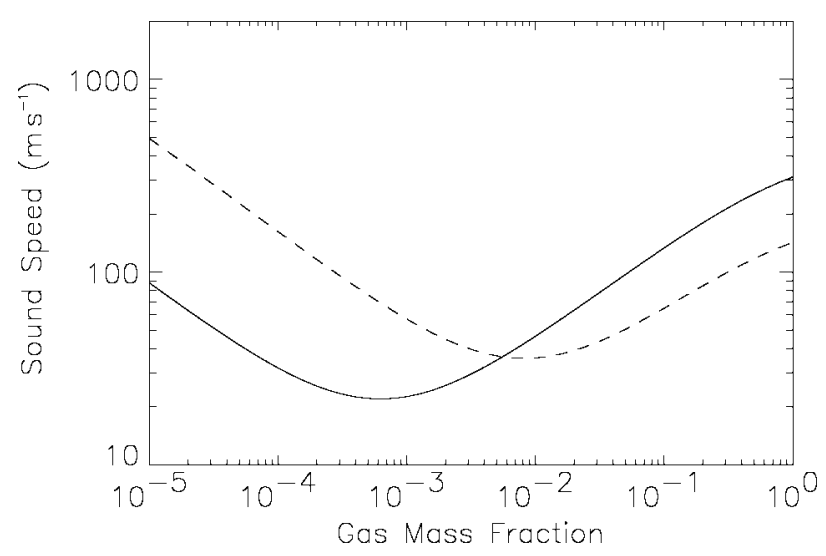

FIG. 4. Dependence of sound speed of a two-phase mixture on mass fraction. The minimum sound speed for water-steam occurs at mass fractions less than a thousandth, whereas around $1 \%$ of the liquid must boil to reach the (higher) minimum speed for Titan fluids (dashed line). 


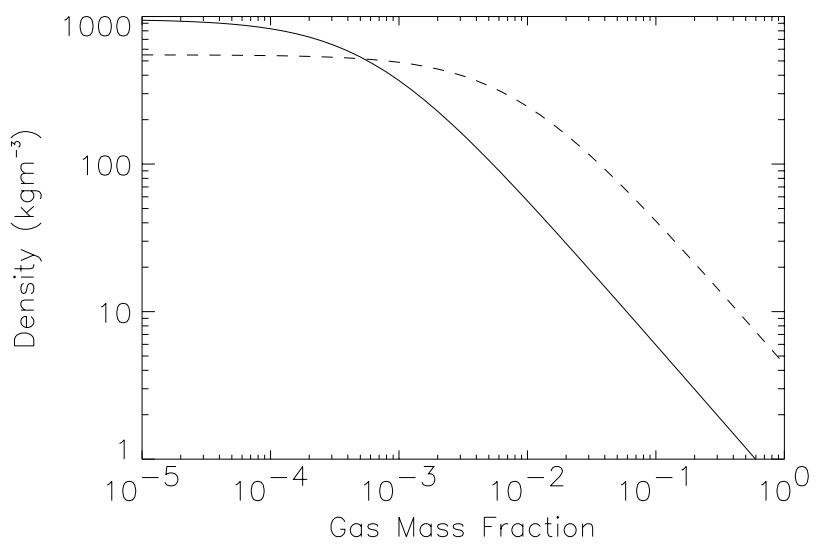

FIG. 5. Density of the two-phase fluid as a function of gas mass fraction. The drop in density (and thus increase in volume) for steam (solid line) is much more significant, occurring at a mass fraction 10 times lower than for Titan (dashed line).

Methane on Titan is much closer to its critical point than is water on Earth. Thus the ratio of densities of steam to liquid on Titan is higher than that on earth. Specifically, the ratios is about 0.01 for Titan but 0.001 for Earth. Hence a given steam mass fraction on Titan will yield a volume increase that is much smaller than that for Earth. To double the volume of a watersteam column on Earth, only $0.1 \%$ of it must boil; yet fully $1 \%$ must boil on Titan to achieve the same effect. Figure 5 plots the fluid density as a function of gas mass fraction.

Considering our canonical 10-m geyser root, with $\Delta T=20 \mathrm{~K}$ for Earth, one obtains $f=3.6 \%$. The corresponding sound speed is around $60 \mathrm{~m} \mathrm{~s}^{-1}$, somewhat higher than the minimum value of around $25 \mathrm{~m} \mathrm{~s}^{-1}$. Where the steam fraction is lower than this, the sound speed will be close to the minimum-in reasonable agreement with the assertion of Kieffer (1977) that geyser flow is typically choked.

For Titan, $\Delta T=3 \mathrm{~K}$ and $f=0.018$. This yields a minimum sound speed for the relevant fluid of approximately $40 \mathrm{~m} \mathrm{~s}^{-1}$. This value is rather larger than that corresponding to the Carnot limit introduced in the previous section; in other words, flow on Titan is limited more by available energy than by hydrodynamic constraints.

The sound speed is not a strict limit for the eruption velocity, in that the velocity downstream of the throat in a choked flow will accelerate (and hence the usefulness of convergent-divergent or de Laval nozzles in rocket motors.) Continuity of mass requires that the product $A \rho u$ where $u$ is the speed, $\rho$ the local fluid density, and $A$ the cross-sectional area, be constant. Since the throat must be the narrowest point, if the fluid were incompressible, the flow must everywhere be less than the speed at the throat, where $u=c$. However, if the liquid can expand after the throat, the velocity will increase in approximate proportion to the specific volume of the fluid. However, estimating how much expansion occurs as a function of time or equivalently position along the duct requires detailed modeling for which there are few good constraints, even on Earth. In general, the eruption velocity will be within a small factor of the sonic limit.

\subsection{Losses and Geyser Height}

The above calculations provide estimates of the maximum possible eruption velocity and corresponding ballistic heights for a 10-m-deep geyser duct. The superheat associated with 10-m depth has been used as a benchmark; clearly deeper roots and hence higher superheats will provide more energetic and efficient eruptions. However, frictional loss along the walls of the duct will be more significant.

More importantly, geyser eruptions are not quite ballistic. Just as a waterfall breaks into a plume of droplets, the jet from a geyser will tend to diffuse into a cloud of drops. It is difficult to model these effects, although some insight can be obtained by considering the terminal velocity of slugs of liquid.

Crudely, a 1-m cube of water has a terminal velocity of order $100 \mathrm{~m} \mathrm{~s}^{-1}$ and the velocity scales as the square root of dimension. Ultimately a column of water would break up into a plume of drops whose size is determined by the balance of surface tension and aerodynamic forces; raindrops are governed by the same balance and have a typical maximum size of $6 \mathrm{~mm}$ with a terminal velocity of $9 \mathrm{~m} \mathrm{~s}^{-1}$. On Titan the same calculation yields a terminal velocity of $12 \mathrm{~m} \mathrm{~s}^{-1}$ for a $1-\mathrm{m}$ cube: The raindrop limit (Lorenz 1993) is 9-mm diameter, with a terminal velocity of $1.6 \mathrm{~m} \mathrm{~s}^{-1}$.

Thus, as might be qualitatively expected from Titan's thick atmosphere and low gravity, a Titan geyser will be more rapidly braked by the atmosphere, and hence the geyser height for a given eruption velocity will be reduced more than on Earth. Nonetheless, the ballistic height for a $1-\mathrm{m}$ cube braked to $12 \mathrm{~m} \mathrm{~s}^{-1}$ is still some $60 \mathrm{~m}$ in Titan's low gravity. More spectacularly, the drops into which such a plume would break would take close to a minute to fall back to the surface. (In comparison, a terrestrial geyser, limited to $25 \mathrm{~m} \mathrm{~s}^{-1}$ by throat sound speed rather than terminal velocity, would reach $30 \mathrm{~m}$ but would fall back as drops in only a few seconds.)

\section{GEYSERING INTERVAL}

Assuming that a geyser is limited by the energy flow required to precipitate an eruption, rather than liquid recharge limitations, the time between eruptions can simply be written as $t=$ $z \rho c_{p} \Delta T / 2 G F$, where $F$ indicates the factor by which the heat flow expressed by the geyser is concentrated from the surrounding region. For our canonical terrestrial geyser, placed say in Yellowstone with $G=4 \mathrm{~W} \mathrm{~m}^{-2}, z=10 \mathrm{~m}, \Delta T=20 \mathrm{~K}, \rho=$ $1000 \mathrm{~kg} \mathrm{~m}^{-3}$, and $c_{p}=4200 \mathrm{~J} \mathrm{~kg}^{-1} \mathrm{~K}^{-1}$, we find $t=10^{8} / F$. For geysering intervals of order tens of minutes (typical of two popular contemporary geysers, Old Faithful and Strokkur), $F$ is therefore around $10^{5}$. In essence this means that a geyser with a duct area of around $1 \mathrm{~m}^{2}$ collects heat from an area some hundreds of meters across. 
Interestingly, if we apply the same calculation to Titan, the same order of magnitude appears; if $F$ is a similar value, the interval is again of order $10^{3} \mathrm{~s}$ (because the required superheat for eruption with methane is lower in more or less equal proportion to the lower heat flow on Titan).

\section{EXOTIC GEYSERING MECHANISMS ON TITAN}

Since geysering can be triggered by unloading of a column, rather than heating of it, tides may play a role. (Tides do appear to affect geyser intervals on Earth, via tidal stress on the surrounding rock affecting the recharge rate; see Rinehart (1980).) The equilibrium tidal potential exerted by Saturn on Titan, expressed as a height, is $100 \mathrm{~m}$, some $400 \times$ larger than that exerted by the Moon on the Earth. However, since Titan rotates synchronously, pointing the same face toward Saturn, this tidal bulge is, to a first order, static. Second-order effects due to Titan's nonnegligible eccentricity $(e=0.029)$ are significant, however. First, the change of distance from Saturn causes a diurnal $3 e(=9 \%)$ variation in tidal potential: If there were a global ocean on an undistorted (spherical) Titan, the 100-m tidal bulge would go up and down by 9 m every 16-day orbit (Sagan and Dermott 1982). Second, while Titan rotates at a constant rate, the vector to Saturn varies in angular rate according to Kepler's second law, and thus the location of Saturn in the sky seen from Titan (and thus the direction of the long axis of the tidal bulge) rocks back and forth by about $3^{\circ}$.

It is known from radar (Muhleman et al. 1995), from nearinfrared imaging from the Hubble Space Telescope (Smith et al. 1996 ), and from ground-based telescopes using speckle and adaptive optics techniques that Titan is not covered in a global ocean. Many smaller scale lakes and seas are the probable explanation of dark features seen in these images, and these may be up to around $600 \mathrm{~km}$ in extent. Unless large channels connect these seas, they do not respond to the varying tidal potential in a manner that would lead to significant tidal dissipation (Dermott and Sagan 1995).

However, lack of large-scale dissipation does not mean an absence of significant tidal effects (just as the lack of a global ocean does not rule out widespread lakes and seas). Thus, imagine a narrow geothermally heated column in the intertidal zone of a hydrocarbon sea. As the tide falls, the load on the liquid column drops and this might stimulate an eruption if the column were on the threshold of boiling. A $600-\mathrm{km}$ lake in the "ideal spot" on Titan (specifically $45^{\circ}$ from the sub- or anti-Saturn points) would have a tidal amplitude of $2 \mathrm{~m}$, or equivalently 10 mbar.

Another, more exotic, mechanism involves the tide in Titan's atmosphere (Lorenz 1992). Again assuming a rigid spherical surface, the surface pressure varies from place to place by about 7 mbar due to the influence of Saturn's gravity. This tidal bulge also varies in amplitude by about $9 \%$ throughout a Titan day. (Thermal tides are likely to be larger than gravitational tides in the atmosphere, as on Earth and Mars, but modeling more elab- orate than justified for this paper would be required to evaluate these.)

Of course, both of the above mechanisms are partially suppressed if Titan has a soft interior and thus the shape of the solid planet itself responds to the tidal potential. However, if Titan is weak like this with a thin lithosphere above a relatively near-surface liquid mantle at $176 \mathrm{~K}$ (see e.g., Lorenz 1996) the geotherm on Titan should be quite steep, which favors the occurrence of conventional geysers.

Solar-thermal geysers, analogous to those on Triton, are also conceivable. Although the insolation at Titan's surface is considerably attenuated due to absorption by haze and methane, about $10 \%$ of the sunlight incident on Titan reaches the surface. This flux of around $1.5 \mathrm{~W} \mathrm{~m}^{-2}$ at high noon, is orders of magnitude higher than the geothermal flux on Titan, and hence it may be significant.

To be effective in promoting geysers, this sunlight must be absorbed at depth, rather than at the surface. However, the spectrum of light reaching the surface has already been filtered by the atmospheric haze, leaving only red and infrared light, and by atmospheric methane, which eliminates light in near-IR methane bands. Thus the light reaching the surface is weakly absorbed by methane and so will penetrate many meters into the liquid surface. Such geysers would probably preferentially erupt in early afternoon, where insolation is highest and can penetrate more steeply into the depths of the geyser pool.

Such warming may still be too strongly coupled to the surface by convection to prompt geysering; instead surface boiling or evaporation may be enhanced. However, surface evaporation might have the effect of unloading a slowly recharged column and stimulating a marginal geothermally heated geyser.

It is possible to conceive of Lake-Nyos-style gas-driven geysers, if lakes on Titan have gas fluxes into their bases that could lead to supersaturation of that gas, bubble formation, and overturn. However, the most likely gas to be released from Titan's interior is probably methane, which is not above its boiling point at Titan and so would not drive such an eruption. Nitrogen, carbon monoxide, helium, and argon would work, but they are unlikely to be supplied in abundance from Titan's interior.

\section{EXPECTATIONS FOR CASSINI}

The possibility of methane geysers has long been recognized, given our ignorance of Titan's surface, and this paper has only established that possibility on physical grounds. Actual detection of geysers is another matter entirely. Historically on Earth, apart from the specific locales in which geysers occur, reports of them were generally greeted with incredulity. The Cassini spacecraft will make around 44 flybys of Titan, with typical observation resolutions of a few hundred meters (perhaps better for the Imaging Science Subsystem, although this depends on the contrast through Titan's thick haze). At such resolution it might be possible to detect geyser plumes and, by nearand far-infrared spectroscopy, local enhancements in methane 
abundance. Spectroscopy would be able to locate such plumes as being at low altitude. Microwave radiometry with a resolution of $0.1 \mathrm{~K}$ should be sensitive to the enhancements of subsurface temperature gradient required for geysering, since microwaves have a penetration depth of order a few meters. Careful analysis would be required to eliminate ambiguities due to composition effects, however. The combination of near-surface plumes and a geothermal anomaly would be compelling, but perhaps not conclusive, evidence of geyser activity. Short of fortuitous proximity to the descent path of the Huygens probe, direct observation of geyser activity would have to wait until future exploration of Titan by mobile platforms such as airships; early plans for such missions are presently under study (Lorenz 2000).

\section{CONCLUSIONS}

Classic and gassy geyser eruption styles on Earth have been considered, together with other related phenomena in the Solar System. Crude constraints on geyser eruption have been expressed, and the conditions for geysers on Titan have been established. Eruption velocities seem constrained more by available energy and the poor conversion efficiency mandated by the small temperature contrasts permitted by the volatile working fluid; this limitation seems stronger than the sound-speed limitation typical for terrestrial geysers.

Although immediate prospects for detecting geysers on Titan seem modest, a Titan geyser would be quite spectacular to an immediate observer, for it would blast quickly to a height rather greater than those of terrestrial geysers (owing to the low gravity), with the plume hanging for some time in the dense air.

\section{ACKNOWLEDGMENTS}

The author acknowledges the support of the Cassini project. This paper was inspired in part by the LPL class field trip to Yellowstone National Park in September 1997 and by the field trip associated with the 2nd International Conference on Mars Polar Science and Exploration in Reykjavik, August 2000. Referees Bonnie Buratti and John Dowden are thanked for useful comments.

\section{REFERENCES}

Anderson, L. W., J. W. Andereff, and J. E. Lawler 1978. Model geysers. Am. J. Science 278, 725-738.

Clarke, T. 2001. Taming Africa's killer lake. Nature 409, 554-555.

Dermott, S. F., and C. Sagan 1995. Tidal effects of disconnected hydrocarbon seas on Titan. Nature 374, 238.

Dowden, J., P. Kapadia, G. Brown, and H. Rymer 1991. Dynamics of a geyser eruption. J. Geophys. Res. 96, 18,059-18,071.

Duxbury, N. S., and R. H. Brown 1997. The role of an internal heat source for the eruptive plumes on Triton. Icarus 125, 83-93.
Garry, J. R. C. 1996. Surveying Titan Acoustically. M.Sc. dissertation, University of Kent at Canterbury.

Kieffer, S. W. 1977. Sound speed in liquid-gas mixtures: Water-air and watersteam. J. Geophys. Res. 82, 2895-2904.

Kieffer, S. W. 1982. Dynamics and thermodynamics of volcanic eruptions: Implications for the plumes on Io. In Satellites of Jupiter (D. Morrison, Ed.), pp. 647-723. Univ. of Arizona Press, Tucson.

Kirk, R. L., L. A. Soderblom, R. H. Brown, S. W. Kieffer, and J. S. Kargel 1995. Triton's plumes: Discovery, characteristics and models. In Neptune and Triton (D. Cruikshank, Ed.), pp. 949-990. Univ. of Arizona Press, Tucson.

Kouvaris, L. C., and F. M. Flasar 1991. Phase equilibrium of methane and nitrogen at low temperatures: Application to Titan. Icarus 91, 112-124.

Lorenz, R. D. 1992. Gravitational tide in the atmosphere of Titan. In Proceedings of the Symposium on Titan, Toulouse, September 1991, ESA SP-338, pp. 119 123. European Space Agency, Noordwijk.

Lorenz, R. D. 1993. The life, death and afterlife of a raindrop on Titan. Planet. Space Sci. 41, 647-655.

Lorenz, R. D. 1996. Pillow lava on Titan: Expectations and constraints on cryovolcanic processes. Planet. Space Sci. 44, 1021-1028.

Lorenz, R. D. 2000. Post-Cassini exploration of Titan: Science rationale and mission concepts. J. Br. Interplanet. Soc. 53, 218-234.

Lorenz, R. D., and S. E. Shandera 2001. Physical properties of ammonia-rich ice: Application to Titan. Geophys. Res. Lett. 28, 215-218.

Lorenz, R. D., C. P. McKay, and J. I. Lunine 1999. Analytic investigation of climate stability on Titan: Sensitivity to volatile inventory. Planet. Space Sci. 47, 1503-1515.

Lorenz, R. D., J. I. Lunine, C. P. McKay, and P. G. Withers 2001. Entropy production by latitudinal heat flow on Titan; Mars and Earth. Geophys. Res. Lett. 28, 415-418.

McKay, C. P., J. B. Pollack, J. I. Lunine, and R. Courtin 1993. Coupled atmosphere-ocean models of Titan's past. Icarus 102, 88-98.

Muhleman, D. O., A. W. Grossman, and B. J. Butler 1995. Radar investigations of Mars, Mercury and Titan. Annu. Rev. Earth Planet. Sci. 23, 337-374.

Rinehart, J. S. 1980. Geysers and Geothermal Energy. Springer-Verlag, Berlin.

Sagan, C., and S. F. Dermott 1982. The tide in the seas of Titan. Nature 300, 731-733.

Smith, P. H., M. T. Lemmon, R. D. Lorenz, J. J. Caldwell, M. D. Allison, and L. A. Sromovsky 1996. Titan's surface, revealed by HST imaging. Icarus 119 , 336-349.

Soderblom, L. A., S. W. Kieffer, T. L. Becker, R. H. Brown, A. F. Cook, II, C. J. Hansen, T. V. Johnson, R. L. Kirk, and E. M. Shoemaker 1990. Triton's geyser-like plumes: Discovery and basic characterization. Science 250, 410 415.

Thompson, W. R. 1985. Phase equilibria in $\mathrm{N}_{2}$-hydrocarbon systems: Applications to Titan. In The Atmospheres of Saturn and Titan, Proceedings of the International Workshop, Alpbach, Austria, 16-19 September 1985, ESA SP-241, pp. 109-119. European Space Agency, Noordwijk.

Thompson, W. R., J. A. Zollweg, and D. H. Gabis 1992. Vapor-liquid equilibrium thermodynamics of $\mathrm{N}_{2}+\mathrm{CH}_{4}$ : Model and Titan applications. Icarus 97, 187199.

Wood, A. B. 1941. A Textbook of Sound. Bell, London.

Yung, Y. L., M. Allen, and J. P. Pinto 1984. Photochemistry of the atmosphere of Titan: Comparison between model and observations. Astrophys. J. Suppl. 55, 465-506.

Zhang, Y. 1996. Dynamics of $\mathrm{CO}_{2}$-driven lake eruptions. Nature 379, 57-59. 